\title{
The visual white matter connecting human area prostriata and the thalamus is retinotopically organized
}

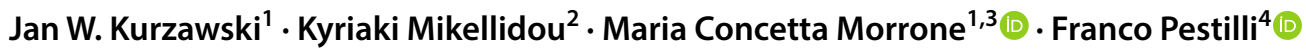

Received: 20 April 2019 / Accepted: 5 June 2020 / Published online: 13 June 2020

(c) The Author(s) 2020

\begin{abstract}
The human visual system is capable of processing visual information from fovea to the far peripheral visual field. Recent fMRI studies have shown a full and detailed retinotopic map in area prostriata, located ventro-dorsally and anterior to the calcarine sulcus along the parieto-occipital sulcus with strong preference for peripheral and wide-field stimulation. Here, we report the anatomical pattern of white matter connections between area prostriata and the thalamus encompassing the lateral geniculate nucleus (LGN). To this end, we developed and utilized an automated pipeline comprising a series of Apps that run openly on the cloud computing platform brainlife.io to analyse 139 subjects of the Human Connectome Project (HCP). We observe a continuous and extended bundle of white matter fibers from which two subcomponents can be extracted: one passing ventrally parallel to the optic radiations $(\mathrm{OR})$ and another passing dorsally circumventing the lateral ventricle. Interestingly, the loop travelling dorsally connects the thalamus with the central visual field representation of prostriata located anteriorly, while the other loop travelling more ventrally connects the LGN with the more peripheral visual field representation located posteriorly. We then analyse an additional cohort of $10 \mathrm{HCP}$ subjects using a manual plane extraction method outside brainlife.io to study the relationship between the two extracted white matter subcomponents and eccentricity, myelin and cortical thickness gradients within prostriata. Our results are consistent with a retinotopic segregation recently demonstrated in the OR, connecting the LGN and V1 in humans and reveal for the first time a retinotopic segregation regarding the trajectory of a fiber bundle between the thalamus and an associative visual area.
\end{abstract}

Keywords Prostriata $\cdot$ Lateral geniculate nucleus (LGN) $\cdot$ Thalamus $\cdot$ Tractography $\cdot$ Myelin $\cdot$ Cortical thickness $\cdot$ Fovea $\cdot$ Periphery

Electronic supplementary material The online version of this article (https://doi.org/10.1007/s00429-020-02096-5) contains supplementary material, which is available to authorized users.

Franco Pestilli

franpest@indiana.edu

1 IRCCS Stella Maris, Viale del Tirreno, 331, Pisa, Italy

2 Department of Psychology and Center for Applied Neuroscience, University of Cyprus, Nicosia, Cyprus

3 Department of Translational Research On New Technologies in Medicine and Surgery, University of Pisa, Pisa, Italy

4 Department of Psychological and Brain Sciences, Program in Neuroscience and Program in Cognitive Science, Indiana University, 1101 E 10th Street, Bloomington, IN 47401, USA

\section{Introduction}

Mikellidou et al. (2017b) described for the first time the functional properties of area prostriata in humans using a novel wide-field visual stimulation system (Greco et al. 2016). In humans, area prostriata is located at the junction between the calcarine and parieto-occipital (POS) sulci, adjacent to the far peripheral representation of $\mathrm{V} 1$ and ventral V2 (Mikellidou et al. 2017b). Area prostriata preferentially responds to very fast motion, greater than $500 \%$, and has a complete representation of the visual field, clearly distinct from the adjacent area V1. Interestingly, area prostriata shows little, if any, cortical magnification but rather has a homogeneous distribution of receptive field centres across eccentricity. The posterior-dorsal border of human prostriata-abutting V1-represents the far peripheral visual field, with eccentricities decreasing toward its anterior boundary in the fundus of the calcarine 
sulcus. This is an anatomical organization comparable to that reported in the common marmoset (Yu et al. 2012). The functional properties of area prostriata suggest that the area may serve to alert the brain about fast visual events, particularly in the peripheral visual field and it could subserve as a critical node for transient attention (Liu et al. 2005).

The cortical position, structural and functional properties of human area prostriata (Ding et al. 2016; Glasser et al. 2016; Sanides 1969) are similar to other species (Kobayashi and Amaral 2003; Morecraft et al. 2000; Rosa et al. 1997; Sanides and Vitzthum 1965). In humans, area prostriata shows lower myelination and increased cortical thickness as compared to neighbouring visual areas (see, Fig. 4f, $g$ in Glasser et al. (2016)). The area shows characteristics akin to those of the limbic cortex (Rockland 2012), as it lacks the clearly defined laminar profile evident throughout the cerebral cortex, with a thinner layer 4 and a thicker layer 2 (Ding et al. 2003). Figure 1 shows coronal and sagittal planes of human area prostriata manually labelled in red on an atlas (1st column) and a histological slice ( 2 nd column).
A T1-weighted slice is also shown with area prostriata in white mapped from a multimodal atlas (3rd column).

As of today, little is known about the anatomical connections of human area prostriata. The connections of area prostriata have been reported in animal models using retrograde and anterograde tracers but to date, these are unknown in the human brain. Prostriata connections, including the motion-sensitive MT + , auditory cortices, cingulate motor areas, the orbitofrontal cortex, and the frontal polar cortices (Burman et al. 2011; Falchier et al. 2010; Morecraft et al. 2000; Palmer and Rosa 2006), support the proposal that it can act as an interface for the coordination of motor responses required in orienting, and could mediate attentional shifts, postural and defensive reactions in 'fight' or 'flight' situations. In humans, it is challenging to establish whether prostriata has a connection (input and/or output) to the thalamus, using the available non-invasive techniques. Recently published work that examined connections of prostriata in rodents showed more evident connections between peripheral V1 and prostriata in comparison to lateral, more foveal $\mathrm{V} 1(\mathrm{Lu}$

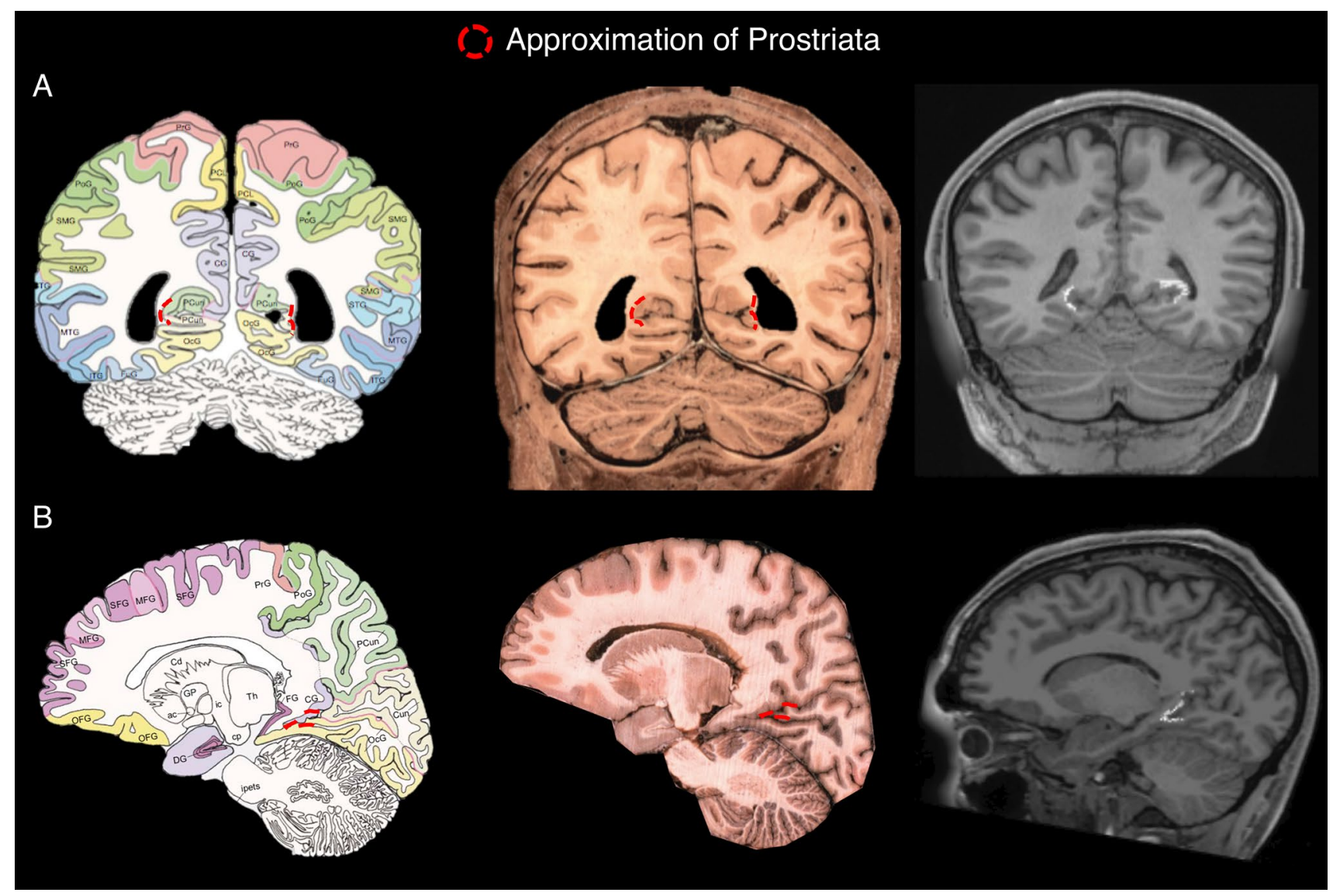

Fig. 1 Putative area prostriata labelled in red trace on a labelled atlas, a histological slice (Mai et al. 2004) and in white on a T1-weighted slice from one of our subjects. In T1-weighted image, prostriata has been mapped from a multimodal parcellation template (Glasser et al. 2016). a Coronal view. b Sagittal view 
et al. 2019). Moreover, Lu et al., proposes that prostriata might be directly connected to the thalamus, as previously suggested (Ding 2013).

Our aim here is to use computational tractography to explore the structural connectivity of human area prostriata with a portion of the thalamus that includes the lateral geniculate nucleus (LGN). In primates and cats, the LGN provides direct input to primary and associative visual cortex, but it has also been shown to receive numerous feedback projections from afferent cortical fibers of the visual cortex (Florence and Casagrande 1987; Sherman and Guillery 2002). To investigate the structural connectivity of area prostriata with the LGN, we combined three different data modalities (T1-weighted, T2-weighted, and functional MRI) with the use of modern tractography, statistical evaluation methods and Human Connectome Project (HCP) data. We performed tractography, evaluated the results using LiFE (Pestilli et al. 2014) and then combined the functionally defined maps, the myelin content and cortical thickness maps together with tractography to fully characterize the corticothalamic connections of area prostriata. To analyse the LGN-prostriata tract, we employ both an automatic procedure based on fiber projections and a manually curated method based on the anatomical trajectory of the tract.

\section{Results}

\section{Estimating eccentricity, myelin content and cortical thickness of area prostriata using public datasets and atlases}

We set to report the relationship between functional and anatomical properties of area prostriata using published datasets and atlases (Mikellidou et al. 2017b; Benson et al. 2018; Glasser et al. 2016). We focused on eccentricity maps (Mikellidou et al. 2017b; Benson et al. 2018), myelin-sensitive maps (Hagiwara et al. 2018; Shams et al. 2019; Glasser and Van Essen 2011) and cortical thickness maps (Glasser et al. 2016). The retinotopic organization of area prostriata estimated using functional neuroimaging shows a typical pattern (Mikellidou et al. 2017b; Yu et al. 2012). It has been reported that the visual field periphery is located in the most posterior region of prostriata, abutting V1 periphery (Fig. 2a). The central portion of the visual field is located in the anterior portion of prostriata. This indicates the presence of a posterior to anterior eccentricity gradient in area prostriata. This reversal is a canonical property of visual field organization in human visual cortex (Wandell and Winawer 2015; Wandell et al. 2005).

We investigated the degree of generality of prostriata's anterior-posterior eccentricity axis using an independent dataset (Glasser et al. 2016). The human brain atlas published by Glasser et al. (2016) contains a map of prostriata (ProS ROI). We show that the location of prostriata estimated by Mikellidou et al. (2017b) is very similar to the one reported by Glasser and colleagues (2016). To do so, we report the barycentre of prostriata in Talairach coordinates as provided by Glasser et al. (2016): $X:-22.0, Y:-55.7, Z: 2$, and compare these coordinates to the barycentre estimated by Mikellidou et al. (2017b) $X:-19.8 \pm 1.7, Y:-60.7 \pm 3.0$, $Z: 1.4 \pm 4.5$. The Talairach coordinates show a high degree of overlap. This is noticeable especially given the differences in mapping method and resolution used in the two studies.

We next investigated whether an anterior-posterior axis can be observed within area prostriata from eccentricity, myelin-sensitive and cortical thickness maps (Fig. 2b-d) using data from the Human Connectome Project (Glasser et al. 2016; Benson et al. 2018). Anterior-posterior axes (map gradients) are visible in each map. First, we replicated the existence of an anterior-posterior eccentricity gradient in the HCP dataset (Fig. 2b). In addition to that, we also show that a similar anterior-posterior axis exists in the myelin-sensitive maps (Fig. 2c) and in the cortical thickness maps (Fig. 2d). In sum, the most anterior part of prostriata processes the central visual field, contains less myelin and has higher cortical thickness, while the posterior part of prostriata processes the peripheral visual field, has higher myelin content and lower cortical thickness. A similar orderly relationship between eccentricity, cortical thickness and myelin content maps has also been previously demonstrated for other visual areas (Abdollahi et al. 2014; Burge et al. 2016). These published data provide an underdescribed anterior-posterior axis in area prostriata consistent across multiple data modalities.

\section{Visual white matter connections between prostriata and the thalamus}

In this section, we set to identify the visual white matter connections between area prostriata and the thalamus. We used multishell diffusion-weighted imaging (DWI) data (Glasser et al. 2013; Andersson et al. 2003; Andersson and Sotiropoulos 2015, 2016) and ensemble tractography (Caiafa and Pestilli 2017; Pestilli 2015; Pestilli et al. 2014; Takemura et al. 2016a; Smith et al. 2012) to track between a subcortical region comprising the LGN and prostriata (see "Methods" for additional details). Tracking results show a large and anatomically complex tract that we refer to as LGN-Pro (Fig. 3a in green).

We note that the tract has a complex anatomical trajectory, with fibers travelling both superiorly and laterally. We also notice that the anatomical trajectory of the fibers within the LGN-Pro tract resembles (but only partially overlaps 


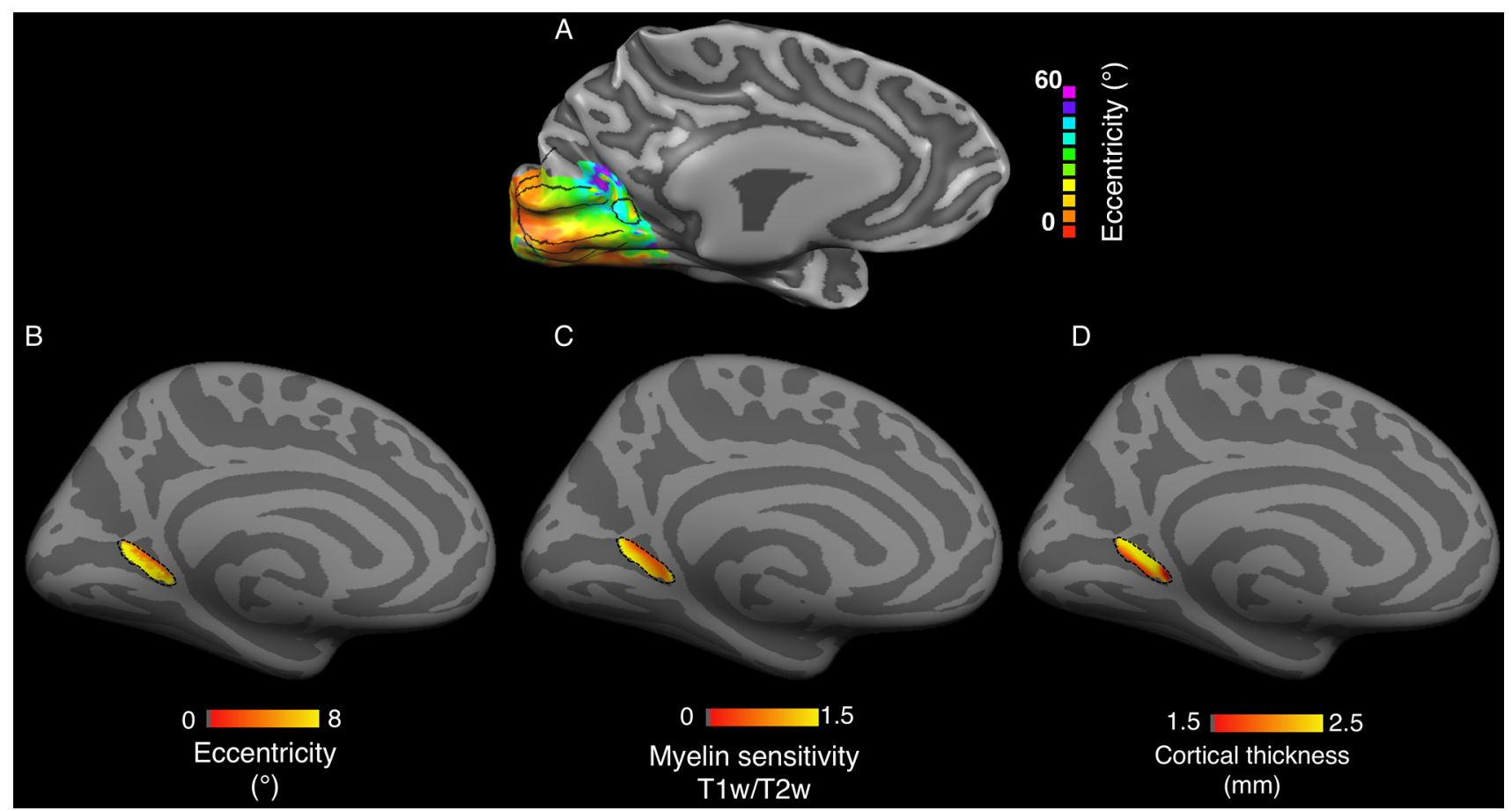

Fig. 2 Structural and functional properties of area prostriata. a Eccentricity representation of subject (S8) from Mikellidou et al., (2017b) with visual field stimulation extending up to 60 degrees. Black lines represent the division between visual areas with a distinct area prostriata located at the fundus of calcarine sulcus. b Eccentric-

with) that of the optic radiations (OR). It has been shown that the OR can be subdivided into at least two subcomponents defined by the termination of the fibers into $\mathrm{V} 1$ regions representing different eccentricities (Parraga et al. 2012; Yoshimine et al. 2018). The fibers of the OR projecting to V1 locations representing the visual periphery travel more ventrally and laterally within Meyer's loop. The fibers of the OR projecting to $\mathrm{V} 1$ locations representing the central visual field travel more dorsally and medially outside Meyer's loop (Yoshimine et al. 2018). Here, we used the anterior-posterior axis of prostriata described in the previous section (Fig. 2) and subdivided the LGN-Pro tract into anteriorprojecting and posterior-projecting fibers (Fig. 3b yellow and cyan respectively). We call the component projecting to anterior prostriata LGN-Pro-1 (yellow) and the component projecting to posterior prostriata LGN-Pro-2 (cyan). These subcomponents were subdivided automatically into 139 subjects using their termination zone in prostriata as a criterion. An LGN-Pro fiber was assigned to LGN-Pro-1 or LGN-Pro-2 depending on whether it terminated into a voxel within the anterior $50 \%$ of the prostriata voxels or the posterior 50\% respectively (Fig. 2). This analysis was performed automatically using brainlife.io (Avesani et al. 2019). To do this, we developed an App (https://doi.org/10.25663/brain life.app.282) that can be publicly accessed and used on new ity representation $(N=181)$ within area prostriata with a visual field stimulation extending up to 8 degrees using HCP data (Benson et al. 2018). c Myelin content and d cortical thickness maps $(N=210)$ within area prostriata created using HCP data (Glasser et al. 2016)

data (see "Methods" for additional details on the full set of Apps on brainlife.io that were used for this analysis).

A density map reports the spatial position of LGN-Pro as a whole, LGN-Pro-1 and LGN-Pro-2 across 139 subjects (11 excluded from 150 batch) aligned to the MNI template (Fig. 3). Figure 3a shows that LGN-Pro is an anatomically continuous bundle across the majority of subjects. Figure $3 b$ compares the spatial position of LGN-Pro-1 (yellow) and LGN-Pro-2 (cyan). Thresholding the map, by showing only the fibers consistently present in at least 90 subjects (65\%), shows that LGN-Pro-1 (yellow) has a dorsal trajectory and LGN-Pro-2 (cyan) has a ventral trajectory. Figure $3 \mathrm{c}$ shows substantial overlap between LGN-Pro-2 and the OR. The exact and detailed separation of LGN-Pro-2 and OR would require other, probably histological, methods. Although the white matter bundle is shared between the two, LGN-Pro-2 originates from prostriata that is clearly different from V1 and therefore we consider it as a separate entity.

Finally, we used the diffusion-tensor model (Basser et al. 1994; Pierpaoli et al. 1996) and estimated fractional anisotropy (FA), mean diffusivity (MD) and radial diffusivity (RD) at 100 locations along the tracts' length using tract profiles (Rykhlevskaia et al. 2009; Yeatman et al. 2012; Yoshimine et al. 2018). The two tracts differ substantially along their length in their microstructural properties (FA, MD, RD; 


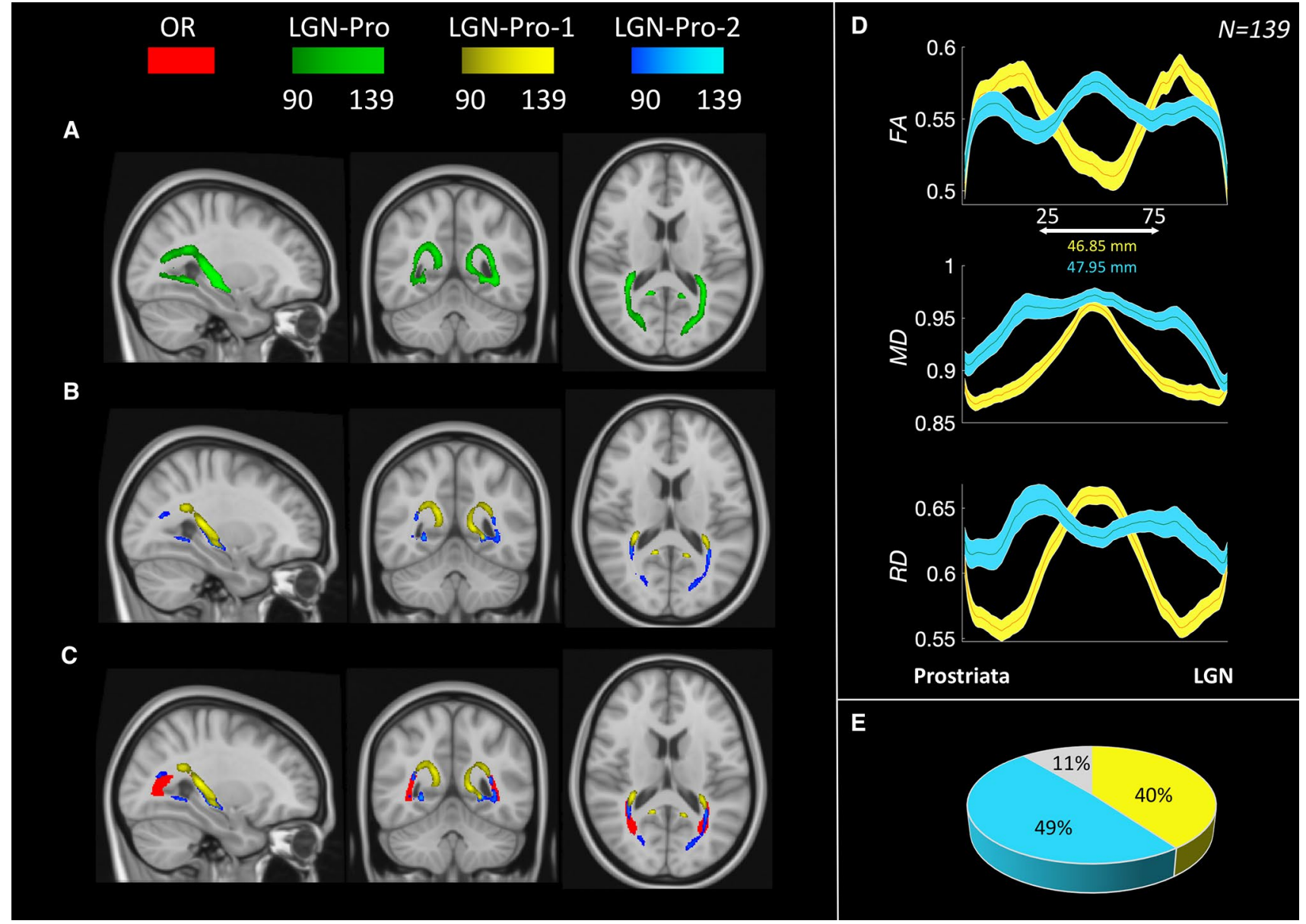

Fig. 3 A. Map of the LGN-Prostriata tract averaged across subjects in the MNI space. B. LGN-Pro-1 (yellow) and LGN-Pro-2 (cyan) subcomponents averaged across subjects in the MNI space. C. LGNPro subcomponents shown alongside the OR (red). The colormap in A, B, C represents consistency across 139 subjects (See "Group averages" in "Methods" for details). D. Diffusion properties along the

Fig. 3d), while the automatic subdivision of subcomponents reveals a similar number of fibers assigned to each of them (Fig. 3e).

\section{The LGN-Pro subcomponents defined using tract trajectory map onto prostriata locations with different properties of eccentricity, myelin-content and thickness}

In the previous section, we characterized the anatomy and microstructure of the complex white matter tract connecting area prostriata and the visual thalamus. We subdivided the tract into two subcomponents based on their cortical locus within prostriata (anterior/posterior). The analysis revealed two subcomponents with different anatomical trajectories and microstructural properties. Here, we investigate whether these two subcomponents show any relationship with tract for both subcomponents. E. Percentage of total fibers between LGN and Prostriata classified as LGN-Pro-1 (yellow) and LGNPro-2 (cyan). In grey we report the percentage of fibers not univocally assigned to either the anterior (foveal, low-myelin, high-cortical thickness) or posterior (peripheral, high-myelin, low-cortical thickness) prostriata regions

eccentricity, myelin and cortical thickness gradient maps within prostriata (Fig. 2), defined using their anatomical trajectories.

To study the relationship between LGN-Pro-1, LGN-Pro2 , and prostriata gradients (eccentricity, myelin-sensitivity and cortical thickness), we extracted two subcomponents using a different method outside brainlife.io on an additional cohort of HCP subjects $(N=10)$. Learning from the anatomical organization of the LGN-Pro tract shown in Fig. 3b-c, we extracted LGN-Pro-1 and LGN-Pro-2 using a manual "plane extraction" method (Zhang et al. 2010; Catani and Thiebaut de Schotten 2008). Specifically, to extract the dorsal subcomponent (LGN-Pro-1), we drew several exclusion planes (available as ROIs) to remove all fibers that travel ventrally. Similarly, to extract LGN-Pro-2 (ventral subcomponent), several exclusion planes were drawn to remove all 

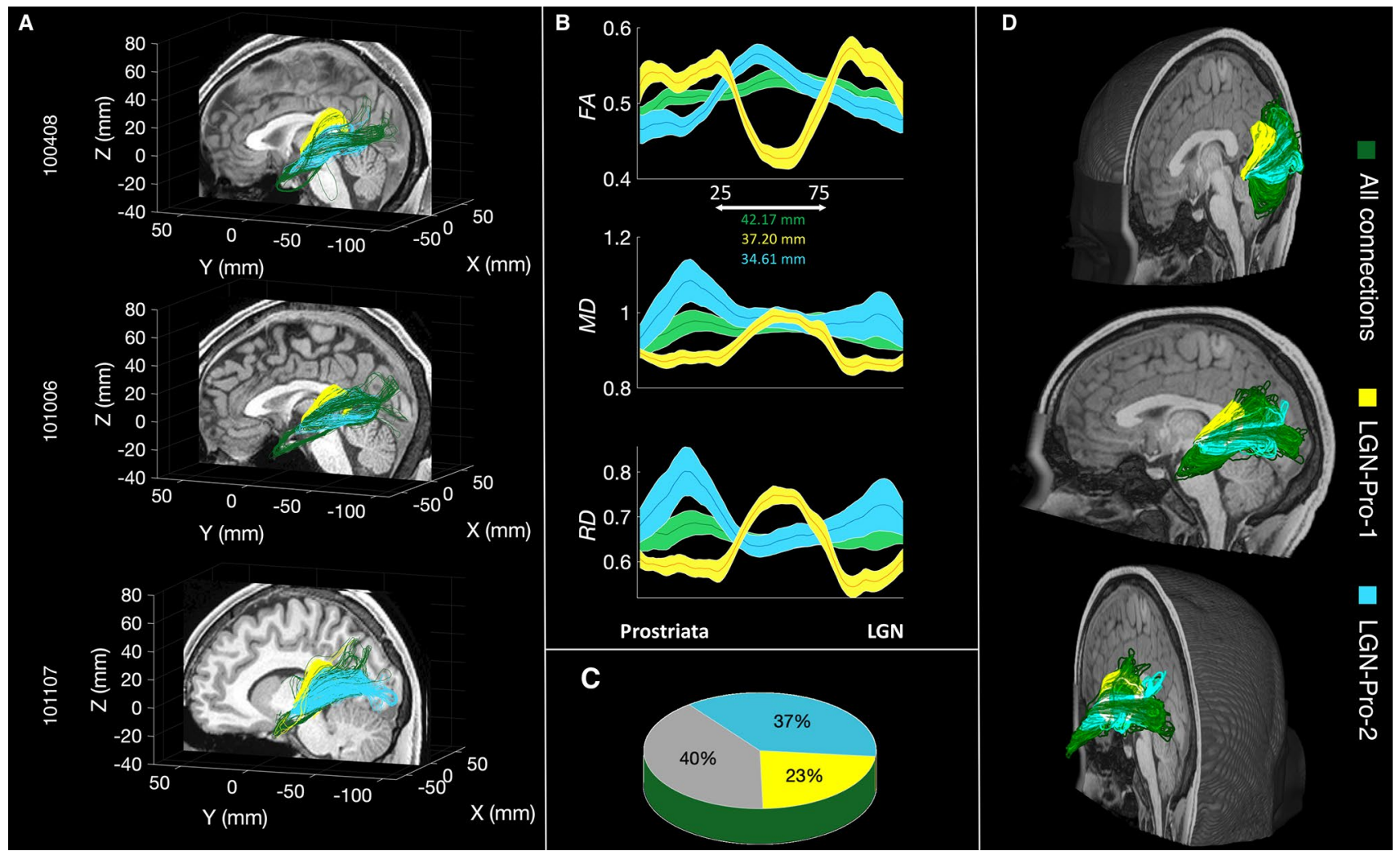

Fig. 4 a A streamline-based representation of the white matter tract between the LGN and prostriata for three example subjects from the HCP dataset (100408, 101006, 100206). One of the two subcomponents of the tract (LGN-Pro-1 in yellow) encompasses the lateral ventricle, reaching the LGN from a superior direction. The second subcomponent (LGN-Pro-2 in cyan) follows a route similar to the OR. b Average diffusion properties (FA, MD, RD) resampled to one hundred points for the tracts displayed in yellow and cyan. The dis-

fibers that travel dorsally (see "Methods" for additional details).

Figure $4 \mathrm{a}$ shows these two subcomponents of the LGN-prostriata tract in three individual subjects: LGN-Pro-1 (yellow) going around the lateral ventricle, reaching the LGN from a superior direction, quite likely through a set of association fibers (the tapetum) and LGN-Pro-2 (cyan) which follows a route similar to that of the OR connecting LGN with V1. On average, the extracted LGN-Pro- 1 comprises $23 \%$ of the fibers in the complete tract, whereas LGN-Pro- 2 comprises $37 \%$ of the total number of fibers connecting LGN with prostriata (pie chart of Fig. 3c, additional right hemisphere images shown in Supplementary Figs. 1 and 2). It is important to note that a large majority of fibers (40\%; Fig. 3a) travel somewhere in between these two subcomponents, indicating that LGN-Pro is a continuous white matter bundle of which we are highlighting two components using a purely anatomically definition. We note that this extraction is somehow arbitrary, and three or more subcomponents could have been extracted tance between 25th and 75th points is $37.20 \mathrm{~mm}$ and $34.61 \mathrm{~mm}$ for LGN-Pro-1 and LGN-Pro-2, respectively $(N=10)$. c Proportion of fibers assigned to LGN-Pro-1 (Yellow) and LGN-Pro-2 (Cyan) using a manually curated segmentation method (plane extraction). Across subjects, the two subcomponents make up $60 \%$ of the LGN-Pro connection, with the remaining $40 \%$ intermingled between the two $(N=10)$. d 3D rendering for the LGN-Prostriata connection for a single subject (101309) viewed from different angles

from this tract. Yet, the two subcomponents we describe below comprise fibers with an interesting relationship between white matter and cortical function and structure.

We further examined the microstructural properties along 100 locations of LGN-Pro-1 and LGN-Pro-2 as in the previous section. Again, the FA, MD and RD differ remarkably for LGN-Pro-1 (yellow), LGN-Pro-2 (cyan) and the complete tract (green). We also estimated the length of the complete tract $(84.35 \pm 12 \mathrm{~mm}$; green), LGN-Pro-1 (74.41 $\pm 7 \mathrm{~mm}$; yellow) and LGN-Pro-2 (69.24 $\pm 19 \mathrm{~mm}$; cyan). These tract profiles show patterns similar to the ones shown by segmenting LGN-Pro-1 and LGN-Pro-2 based on their cortical termination zones (compare profiles shown in Figs. 3 and 4).

Defining LGN-Pro-1 and LGN-Pro-2 independently of prostriata (based on their anatomical trajectories) as performed in this section, allowed us to analyse the distribution of the fiber terminations in prostriata. We next set to characterize any relation between the two tracts and the eccentricity, myelin-sensitivity and cortical thickness 
maps within prostriata. We found that the majority of the segregated fibers follow an eccentricity gradient as shown in the cortical maps (Fig. 2). We then combine the results of our tractography analyses (Fig. 4) with the gradient maps (Fig. 2) to establish whether there is a relation between the white matter subcomponents and prostriata.

Figure 5a shows the cortical projection zones of the two extracted white matter tracts on the cortical surface of three representative subjects (see also Supplementary Fig. 3 for left hemisphere). For each individual, we projected the cortical location of the two subcomponents on top of the gradient maps of prostriata. Qualitatively, LGN-Pro-1 appears to project more anteriorly and LGN-Pro-2 appears project more posteriorly. This division is consistent with all gradients shown within prostriata (Fig. 2). In sum, given that the anterior and posterior parts of prostriata process the foveal and peripheral parts of the visual field respectively, our results suggest that whereas LGN-Pro-1 seems to transfer information from the foveal visual field, LGN-Pro-2 transfers information from the periphery. This is also consistent with the higher FA values within prostriata for LGN-Pro-1 (Fig. 4b), also observed in a previous report regarding the OR retinotopic segregation (Yoshimine et al. 2018).
Finally, to provide a quantification of the qualitative results reported above, we divided the prostriata ROI into two parts using the major (longer) axis of the ellipse illustrated in one representative subject in Fig. 5b (this analysis is akin to that performed on 139 subjects for Fig. 3). The gradient which runs along the eccentricity, myelin and cortical thickness maps was used as a guideline to divide prostriata into anterior and posterior components (see "Methods" for details). We then counted the number of fibers entering either the anterior or posterior part of prostriata. We count as 'Hits' the proportion of fibers from the whole LGN-prostriata tract (in green-Fig. 4a) which end in the anterior part of prostriata and are identical to those in LGN-Pro-1. The proportion of fibers from the whole LGN-prostriata tract which pass through the posterior part of prostriata and are identical with those in LGN-Pro-1, are labelled as 'False Alarms'. We compute d-prime (d') which is the standardized difference between the means of the hits and the false alarms. In this case, d' is calculated to 1.90 (hits: $90.1 \%$, false alarms: $26.9 \%$ ). Then we consider as 'Hits' the proportion of fibers from the green tract which pass through the posterior part of prostriata and are identical with those in LGN-Pro-2. The proportion of fibers from the whole LGN-prostriata tract which pass through the anterior part of prostriata and

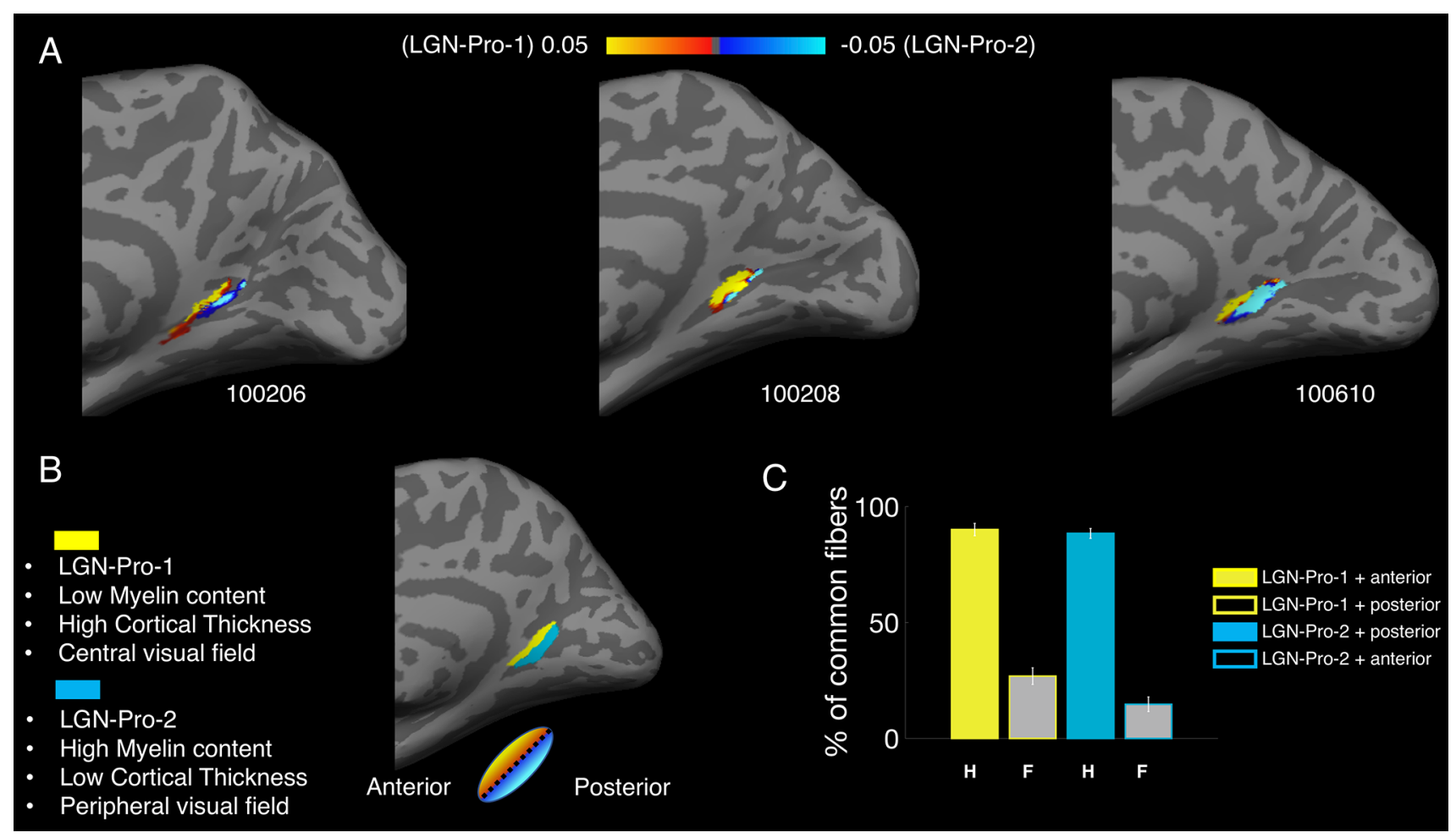

Fig. 5 a Cortical projections of LGN-Pro-1 and LGN-Pro-2 (fiber density distribution in the right hemisphere of three representative subjects). The yellow map corresponds to LGN-Pro-1 which traverses the ventricle and the blue map corresponds to the LGN-Pro-2 which follows the OR. b Schematic summary of the LGN-Pro divi- sion based on the gradient maps. c Overlap between LGN-Pro-1 and LGN-Pro-2. The percentage of total fibers across hemispheres separated into hits (H-colored bars) and false alarms (F-grey bars) for LGN-Pro-1 (yellow face/edge color) and LGN-Pro-2 (cyan face/edge color) which was used to calculate d' prime 
are identical with those in LGN-Pro-2 is considered 'False Alarms'. In this case, d' is calculated to 2.24 (hits: $88.5 \%$, false alarms: $14.7 \%$ ). Results are displayed in the bar plot of Fig. $5 \mathrm{c}$ and demonstrate that the two subcomponents of our tract could indeed be distinguished based on their anatomical pathway.

In summary, we conclude that the anterior part of prostriata with less myelin and high cortical thickness processes information from the central visual field and is connected with the thalamus via the dorsal white matter subcomponent of the tract (LGN-Pro-1). On the other hand, the posterior part of prostriata with increased myelin and low cortical thickness processes information from the peripheral visual field and is connected with the LGN via the ventral subcomponent of the tract (LGN-Pro-2). More interestingly, a similar retinotopic organization of fibers is observed in the thalamus. A retinotopic organization within LGN has been previously reported, with a foveal-peripheral gradient running from medial to lateral direction (Arcaro et al. 2015). We observe a similar organization while examining the endpoints of LGN-Pro-1 and LGN-Pro-2 within our LGN. Three example subjects are shown in Supplementary Fig. 3a where LGN-Pro-1 appears to reach more medial parts and LGN-Pro-2 reaches more lateral parts of the LGN. In Supplementary Fig. 4b, we show a histogram with the $\mathrm{X}$ coordinate of the endpoints in MNI space of each subcomponent from both hemispheres of all participants (lower values-medial, higher values-lateral).

\section{Discussion}

The aim of this study was to explore the structural connectivity of area prostriata in the human brain with the thalamus using diffusion tractography and freely available datasets from the HCP. We observe a white matter bundle connecting area prostriata with the LGN from which two subcomponents can be extracted; one that follows a similar route to the optic radiations and another one that bends around the lateral ventricle and reaches the LGN from a superior direction. We report that the fibers comprising LGN-Pro-2 are placed more posteriorly in prostriata, compared to those comprising LGN-Pro-1 which are more anterior. When comparing our anatomical results with eccentricity maps from previous reports (Mikellidou et al. 2017b; Yu et al. 2012), we observe that the peripheral part of prostriata is connected with the LGN through LGN-Pro-2 following a similar path to the $\mathrm{OR}$, whereas the central visual field representation is connected through LGN-Pro-1 which circumvents the lateral ventricle. The division of the bundle into fibers that transfer information from different parts of the visual fields is also confirmed by the analysis of endpoints within the LGN. LGN-Pro-1 reaches the most medial part of LGN, which has been shown to process the foveal part of the visual field, whereas LGN-Pro-2 innervates more lateral parts, typically processing the visual periphery. Considering the resolution of data, the size of the LGN and limitation of tractography further validation would be necessary. The results obtained with the manual (Figs. 4, 5) and automated method (Fig. 3) are consistent and taken together they demonstrate that it is possible to subdivide the LGN-prostriata white matter tract into two subcomponents, carrying information about different parts of the visual field to different parts of prostriata and the LGN.

This is not the first report of white matter tracts segmented into different subcomponents based on functional properties using in vivo tractography. In fact, the division of a fiber bundle based on visual field representation has been carried out by Yoshimine et al.(2018), (see their Fig. 2) who separate the OR based on eccentricity with clear segregation of foveal and peripheral tracts (Yoshimine et al. 2018; Parraga et al. 2012). We note that the subdivision between LGN-Pro-1 and LGN-Pro-2 is somehow anatomically arbitrary. This is because the bundle appears as a single, contiguous fiber set (Fig. 3a green). Yet, it is interesting to note that retinotopically organized components can be extracted from the LGN-prostriata white matter tract similarly to what has been demonstrated for the OR.

Interestingly, recent evidence shows a white matter bundle of similar shape to LGN-Pro-1 which is encompassing the ventricle, crossing the splenium and extending to the lateral occipital lobe (Yakar et al. 2018). This white matter bundle might belong to the lateral part of the tapetum, descending to the temporal lobe near the OR and the IFOF. Although prostriata is located medially in the occipital lobe, such evidence shows that the tapetum might be able to transfer information between the occipital lobe and other structures. Original work that examined the agenesis of the corpus callosum, argued that the tapetum is a separate structure and defined it as a direct continuation of the occipital frontal bundle (Onufrowicz 1887). However, subsequent work demonstrated that the tapetum is part of the splenium (Schmahmann and Pandya 2007), it consists of callosal fibers, transfers information between hemispheres (Veltin 2003) and it has been delineated in tractography studies (Martino et al. 2013; Tax et al. 2014). Irrespective of its independence from the splenium, the tapetum could be transferring visual information to the visual cortex as shown by a diffusion study that placed a seed in the splenium of the corpus callosum (Caspers et al. 2015). Interestingly, a patient with a transient splenium lesion, near the tapetum, has been reported to have a transient visual field loss with no other neurological symptoms (Gunaydin and Ozsahin 2018).

For the second part of the analysis which was performed locally, we improved the anatomical constraints for tractography to avoid false positives. As the aim was to closely 
follow the trajectory of LGN-Pro subcomponents, we manually corrected the white matter mask for each individual hemisphere, so that the final result is limited only within voxels fully consisting of white matter. During the manual correction, we excluded all voxels with partial volume effects that were incorrectly labelled as white matter and were, in fact, grey matter or cerebrospinal fluid. For the larger cohort of subjects analysed on brainlife.io, we did not carry out any manual correction of the white matter mask as our aim was to determine whether subcomponents can be extracted without extensive post-processing corrections.

To map prostriata ROI on the native surfaces, in the second part of analysis, we used a method developed by Fischl et al. implemented in the mri_surf2surf function as part of Freesurfer (Fischl et al. 1999) that is based on cortical folding patterns and does not require additional functional information (unlike MSM-all). Still, we found a great degree of overlap of the projected zones when using MSM-all (Robinson et al. 2014, 2018) or mri_surf2surf (79.4\%), demonstrating that our results are not affected by alignment procedures. The two subregions of prostriata were mapped based on the HCP 7 T dataset (Benson et al. 2018; Glasser et al. 2016). The stimuli presented in the study did not exceed 8 degrees of eccentricity and, therefore, we assume that the posterior part of prostriata is responsible for processing the peripheral and far peripheral information based on the cortical thickness and myelin sensitivity (Abdollahi et al. 2014; Burge et al. 2016). However, also, in this case, it is possible that the central visual field ROI extends and overlaps the abutting area located more dorsally along the POS (Elshout et al. 2018). In fact, the increased cortical thickness and decreased myelin extend in this abutting cortex that could be sharing the same central visual field representation as it is the case between V1, V2 and V3. This could suggest a different interpretation of the two tracts, with the more dorsal tract (LGN-Pro-1) associated with these new and understudied cortical areas. Either way, our results suggest that associative areas have retinotopically organized projections to and/or from the thalamus and this could become important information to interpret some neuropsychological visual deficit associated with focal lesions in the white matter.

There are of course known limitations when using methods such as tractography. The emergence of false positives from tractography is not unheard of regardless of algorithms used for the analysis due to the ambiguity of diffusion MRI data (Maier-Hein et al. 2017), especially when examining structures close to crossing fibers like the sagittal striatum and OR (Goga and Ture 2015). The main weakness of tractography, however, is that it characterizes the axonal pathways based on indirect evidence. The approach is substantially different from the gold standards used in tracttracing which involve physical monosynaptic transport of dyes along the axonal projections. Tracing, however, is only limited to brain areas of easy access for injection and is not applicable to the human brain. There has been substantial work highlighting the potential disagreement between the two methods (Pierpaoli et al. 2001; Basser et al. 1994) even when using sophisticated methods and high-quality data (Thomas et al. 2014). As a result, confirming the existence of any white matter connection, invasive methods and comparative work are mandatory. Indeed, in vivo tractography findings are often complemented with comparisons to anatomical knowledge from post-mortem methods, advancing knowledge and discovery. For a discussion about statistical methods for tractography evaluation see also (Bullock et al. 2019; Pestilli 2015, 2018; Rokem et al. 2017; Rossini et al. 2019; Takemura et al. 2016b). It has been demonstrated that, under certain conditions of scientific rigor, in vivo tractography helps to clarify the organization of white matter anatomy otherwise left unresolved by post-mortem methods (Takemura et al. 2016b, 2017, 2019; Wandell 2016; Sani et al. 2019; Yeatman et al. 2014; Puzniak et al. 2019; Leong et al. 2016). Furthermore, recent examples have appeared where tractography has led the way by shedding light on undescribed anatomy with post-mortem work providing independent validation (Bullock et al. 2019; Kalyvas et al. 2019).

Our results demonstrate for the first time that there is a structural connection between the LGN and area prostriata in the human brain. Whether this connection is carrying an input from the thalamus, thus transferring information to prostriata, cannot be answered with tractography. The LGN-prostriata connection can equally be associated with the numerous feedback projections that arise from V1 and other visual associative areas projecting monosynaptically back to the thalamus in a retinotopic-specific manner. However, electrophysiological studies in animal models do suggest the existence also of a direct thalamic input. One evidence is the high spontaneous activity and short latencies to visual stimulation of prostriata neurons in the marmoset monkey (Yu et al. 2012). In the tree shrew, a direct thalamic projection to the retrosplenial cortex and specifically area prostriata have been demonstrated anatomically with Thawmount autoradiography (Conrad and Stumpf 1975). Whether also in humans a direct structural input between the LGN and prostriata, bypassing V1, exists is still to be demonstrated, but if so, it could be of primary importance in the presence of $\mathrm{V} 1$ lesions as it could be subserving residual visual abilities (Arcaro et al. 2018; Mikellidou et al. 2017a).

The distinct pattern of white matter connections carrying information from different parts of the visual field between human area prostriata and the thalamus observed here is very similar to what has been observed in V1 (Yoshimine et al. 2018). By analogy, it is tempting to suggest that similar to prostriata all associative visual areas have retinotopically organized tracts connecting the thalamus and the cortex, 
demonstrating that cortical organisation extends beyond function.

\section{Methods}

\section{Data from HCP and pre-processing}

Pre-processed diffusion data together with anatomical T1-weighted images (AC-PC aligned) were uploaded to brainlife.io $(N=150)$ or downloaded locally $(N=10)$ from HCP bucket (Andersson et al. 2003; Andersson and Sotiropoulos 2015, 2016; Van Essen et al. 2013). Fiber orientation density function (FOD) was estimated using Constrained Spherical Deconvolution (CSD) model (Tournier et al. 2004) with maximum harmonic order of $L \max =10$. Both CSD and diffusion tensor were processed with mrTrix 3 (Tournier et al. 2019).

\section{Automated pipeline}

\section{Reproducible neuroimaging methods for automated extraction of the LGN-prostriata white matter tracts: analysis of 150 HCP subjects on brainlife.io}

Brainlife.io is a free, publicly funded, cloud-computing platform that allows for developing reproducible neuroimaging processing pipelines and sharing data (Pestilli 2018; Avesani et al. 2019). To analyse the data, we developed a series of steps that are implemented as Docker containers. The pipeline was used to process 150 subjects from the Human Connectome Project (Van Essen et al. 2013). If any of the four bundles (dorsal and ventral branches for both hemispheres) was not reconstructed during the automatic analysis, the subject was removed $(N=11)$. We first uploaded the data on brainlife.io directly from the HCP bucket and used it to align the DWI images to the T1-weighted space. To generate the ROIs (seeds for tractography), we utilized transformation files that were already available in the HCP data (Glasser et al. 2013). The LGN was aligned using a transformation field obtained with FSL and prostriata was transformed using MSM-all registration and workbench commands. We then fit the tensor and estimate the Fiber Orientation Distribution (FOD) which leads to obtaining all the necessary files for ensemble tractography (Takemura et al. 2016a). All generated tracts have been validated with LiFE (Pestilli et al. 2014) and tract subcomponents were extracted with dtiIntersectFibersWithRoi (minimum distance $=2 \mathrm{~mm}$ ). Tract profiles for each subcomponent were extracted and saved (Yeatman et al. 2012). For clarity, all steps are listed in Table 1 and the code for each app is available on github (https://github.com/brainlife). For customized parameters and to perform our step-by-step procedure, please access the apps using the doi links below.

\section{Manual pipeline}

\section{Definition of ROls}

Cortical ROIs were first projected on the average anatomy 'fsaverage' using the template (Glasser et al. 2016) and mapped back to the native cortical space using the mri_surf2 surf. Volume ROIs that were used as masks for tractography were created using mri_surf2vol command for each subject. Subcortical ROIs were mapped back from Talaraich Deamon labels included in the FSL package (Lancaster et al. 2000). In the tractography session, we used the following regions: the lateral geniculate nucleus (LGN) and area prostriata. The LGN ROI was dilated with maskfilter function (mrTrix, npass $=2$ ). Transformations for both cortical and subcortical ROIs were obtained from HCP.

\section{Tractography}

For each pair of ROIs located in the same hemisphere, we estimated possible white matter tracts that represent the anatomical connections between them. Tractography was constrained by the white matter mask; a union mask of two
Table 1 Web-based applications developed for the data analysis on brain-life.io

\begin{tabular}{ll}
\hline Scope of the brainlife.io app & Weblink to the online app \\
\hline $\begin{array}{l}\text { Uploading DWI data together with T1-weighted ac-pc aligned } \\
\text { anatomy on brainlife.io directly from the HCP bucket }\end{array}$ & No app needed \\
Aligning anatomy to DWI & https://doi.org/10.25663/bl.app.68 \\
Mapping ROIs from templates & https://doi.org/10.25663/brainlife.app.288 \\
Fitting the tensor & https://doi.org/10.25663/bl.app.60 \\
Estimating the CSD & https://doi.org/10.25663/brainlife.app.238 \\
Ensemble tractography (LGN-Pros) & https://doi.org/10.25663/brainlife.app.279 \\
Validating tracts with LiFE & https://doi.org/10.25663/bl.app.104 \\
Intersecting the tract with ROI (dorsal/ventral prostriata) & https://doi.org/10.25663/brainlife.app.282 \\
Analysis of tract profiles & https://doi.org/10.25663/brainlife.app.185 \\
\hline
\end{tabular}


ROIs was used as a seed. For each pair, we included only the fibers that traverse through both ROIs. Seed masks were also used as endpoints, ensuring that white matter connections do not simply pass through our ROIs but actually end there. Tractography was performed using mrTrix software and the streamtrack command. For each set of ROIs, the algorithm discovered a maximum of 10,000 fibers with $1,000,000$ trials. To improve the accuracy of our results we used ensembled tractography (Takemura et al. 2016a) which performs the same tracking procedure but changes the minimum allowed curvature to propagate from voxel to voxel. We used four different curvature thresholds $c=[0.25$ $\left.\begin{array}{llll}0.5 & 1 & 2 & 4\end{array}\right] \mathrm{mm}$ and merged the obtained fibers in our final fiber bundles.

\section{Tractography validation and thresholding}

Obtained tracts were validated with LiFE software (Caiafa and Pestilli 2017; Pestilli et al. 2014). The algorithm predicts the diffusion signal using the orientation of the fascicles present in obtained connections and compare it to the acquired MR data. The difference between the two is used to calculate the prediction error. For each voxel, a weight is assigned that describes how each fascicle contributes towards predicting the diffusion model, with 0 signifying maximum error and 1 no error. Fascicles with zero-weights were discarded from the analysis. This procedure was applied to all tracts.

\section{Manual extraction of prostriata subcomponents LGN-Pro-1 and LGN-Pro-2}

For each subject, we used a plane extraction method to extract two subcomponents, by visually inspecting the connections and drawing an exclusion plane (all planes available as Matlab ROIs compatible with mrDiffusion). We focus on extracting two clearly distinct subcomponents of the LGN-prostriata tract: a dorsal and a ventral one. For example, to extract the dorsal subcomponent (LGN-Pro1), we drew several exclusion planes (available as ROIs) to remove all fibers that travel ventrally. Similarly, to extract LGN-Pro-2 (ventral subcomponent) several exclusion planes were drawn to remove all fibers that travel dorsally.

\section{Mapping the starting points}

To visualize the starting points of the two subcomponents of the tract, we first transformed the tractography results to the volume using dtiFiberendpointNifti function (vistasoft) and followed previously described surface mapping procedures from FreeSurfer (mri_vol2vol, mri_vol2surface). This allowed us to locate and evaluate the exact location within the seed from which fibers originate. The normalized density of fibers was calculated by taking the number of fibers passing through a voxel and dividing by the maximum number of fibers within a voxel included in the track. The density map was masked with the prostriata ROI.

\section{Dividing prostriata into anterior and posterior ROIs}

Anterior and posterior parts of prostriata were manually defined using an eccentricity gradient map (Benson et al. 2018) as well as myelin and cortical thickness gradient maps (Glasser et al. 2016) (see Fig. 2a-c). An example of the division of the ROI on a native surface is shown in Fig. 5b. The ROI was divided into the fsaverage space (50\% of anterior vertices and $50 \%$ of posterior vertices) and both anterior and posterior parts were mapped to the native surfaces for the purpose of this analysis.

\section{Dividing the LGN-prostriata tract based on fiber endpoints within prostriata}

To determine whether anatomically defined (manual method) parts of the tract transfer information from different parts of the visual field, we count the proportion of fibers of the LGN-Pro bundle entering anterior or posterior parts of prostriata (see Fig. 5). We defined the fibers passing through the desired ROI using dtiIntersectFibersWithRoi.

\section{Distribution of fibers within the thalamus}

To calculate the distribution of fibers inside the LGN, we transformed all tracts to the MNI space (Avants et al. 2008). We then sort all the voxels inside the LGN from medial to lateral (X coordinate in MNI) and count how many fibers from each of the subcomponents enter the voxel at this specific location using dtiIntersectFibersWithRoi for each voxel.

\section{Group averages}

To create an average map of estimated tracts, we utilized MNI transformation files available in the HCP project (Van Essen et al. 2013). Each 'tck' tract was transformed to nifti using tckmap function from mrtrix3 (Tournier et al. 2019) and further transformed to the MNI space. Due to the direct mapping of each individual fiber to the volume space, we smoothed the map representing each tract with an FWHM $=2 \mathrm{~mm}$. All tracts in the MNI space were binarized and results in Fig. 3 present the mean 'consistency' map where the value in each voxel represents the number of subjects in which the tract is present at that specific point.

Funding This research was supported by the European Union's Horizon 2020 research and innovation programme under the Marie Skłodowska-Curie Grant Agreement 'Peripheality' grant agreement 
no. 797603 (K.M.) and by the ERC Advanced Grants "Spatio-temporal mechanisms of generative perception", grant agreement no. 832813 - GenPercept (M.C) as well as " Temporal context in perception: serial dependence and rhythmic oscillations " funded by the Italian Ministry of Education, University, and Research under the PRIN2017 programme (Grant 2017SBCPZY_02) (M.C). NSF OAC1916518, NSF IIS-1912270, NSF IIS-1636893, NSF BCS-1734853, NIH 1R01EB029272-01, a Microsoft Faculty Fellowship, the Indiana University Areas of Emergent Research initiative "Learning: Brains, Machines, Children" (F.P.). We thank Bradley Caron, Soichi Hayashi, David Hunt for contributing to developing some of the web services on brainlife.io. We thank Craig Stewart, Robert Henschel, David Hancock and Jeremy Fischer for support with jetstream-cloud.org (NSF ACI-1445604).

Open Access This article is licensed under a Creative Commons Attribution 4.0 International License, which permits use, sharing, adaptation, distribution and reproduction in any medium or format, as long as you give appropriate credit to the original author(s) and the source, provide a link to the Creative Commons licence, and indicate if changes were made. The images or other third party material in this article are included in the article's Creative Commons licence, unless indicated otherwise in a credit line to the material. If material is not included in the article's Creative Commons licence and your intended use is not permitted by statutory regulation or exceeds the permitted use, you will need to obtain permission directly from the copyright holder. To view a copy of this licence, visit http://creativecommons.org/licenses/by/4.0/.

\section{References}

Abdollahi RO, Kolster H, Glasser MF, Robinson EC, Coalson TS, Dierker D, Jenkinson M, Van Essen DC, Orban GA (2014) Correspondences between retinotopic areas and myelin maps in human visual cortex. NeuroImage 99:509-524. https://doi.org/10.1016/j. neuroimage.2014.06.042

Andersson JL, Sotiropoulos SN (2015) Non-parametric representation and prediction of single- and multi-shell diffusion-weighted MRI data using Gaussian processes. Neuroimage 122:166-176. https ://doi.org/10.1016/j.neuroimage.2015.07.067

Andersson JLR, Sotiropoulos SN (2016) An integrated approach to correction for off-resonance effects and subject movement in diffusion MR imaging. Neuroimage 125:1063-1078. https://doi. org/10.1016/j.neuroimage.2015.10.019

Andersson JL, Skare S, Ashburner J (2003) How to correct susceptibility distortions in spin-echo echo-planar images: application to diffusion tensor imaging. Neuroimage 20(2):870-888. https://doi. org/10.1016/S1053-8119(03)00336-7

Arcaro MJ, Thaler L, Quinlan DJ, Monaco S, Khan S, Valyear KF, Goebel R, Dutton GN, Goodale MA, Kastner S, Culham JC (2018) Psychophysical and neuroimaging responses to moving stimuli in a patient with the Riddoch phenomenon due to bilateral visual cortex lesions. Neuropsychologia. https://doi.org/10.1016/j. neuropsychologia.2018.05.008

Avants BB, Epstein CL, Grossman M, Gee JC (2008) Symmetric diffeomorphic image registration with cross-correlation: evaluating automated labeling of elderly and neurodegenerative brain. Med Image Anal 12(1):26-41. https://doi.org/10.1016/j.media .2007 .06 .004

Avesani P, McPherson B, Hayashi S, Caiafa CF, Henschel R, Garyfallidis E, Kitchell L, Bullock D, Patterson A, Olivetti E, Sporns O, Saykin AJ, Wang L, Dinov I, Hancock D, Caron B, Qian Y, Pestilli F (2019) The open diffusion data derivatives, brain data upcycling via integrated publishing of derivatives and reproducible open cloud services. Sci Data 6(1):69. https://doi.org/10.1038/ s41597-019-0073-y

Basser PJ, Mattiello J, LeBihan D (1994) MR diffusion tensor spectroscopy and imaging. Biophys J 66(1):259-267. https://doi. org/10.1016/S0006-3495(94)80775-1

Benson NC, Jamison KW, Arcaro MJ, Vu AT, Glasser MF, Coalson TS, Van Essen DC, Yacoub E, Ugurbil K, Winawer J, Kay K (2018) The Human Connectome Project 7 Tesla retinotopy dataset: description and population receptive field analysis. J Vis 18(13):23. https://doi.org/10.1167/18.13.23

Bullock D, Takemura H, Caiafa CF, Kitchell L, McPherson B, Caron B, Pestilli F (2019) Associative white matter connecting the dorsal and ventral posterior human cortex. Brain Struct Funct 224(8):2631-2660. https://doi.org/10.1007/s00429-019-01907-8

Burge WK, Griffis JC, Nenert R, Elkhetali A, DeCarlo DK, ver Hoef LW, Ross LA, Visscher KM (2016) Cortical thickness in human V1 associated with central vision loss. Sci Rep 6:23268. https ://doi.org/10.1038/srep23268

Burman KJ, Reser DH, Yu HH, Rosa MG (2011) Cortical input to the frontal pole of the marmoset monkey. Cereb Cortex 21(8):1712 1737. https://doi.org/10.1093/cercor/bhq239

Caiafa CF, Pestilli F (2017) Multidimensional encoding of brain connectomes. Sci Rep 7(1):11491. https://doi.org/10.1038/s4159 8-017-09250-w

Caspers S, Axer M, Caspers J, Jockwitz C, Jutten K, Reckfort J, Grassel D, Amunts K, Zilles K (2015) Target sites for transcallosal fibers in human visual cortex - a combined diffusion and polarized light imaging study. Cortex 72:40-53. https://doi. org/10.1016/j.cortex.2015.01.009

Catani M, Thiebaut de Schotten M (2008) A diffusion tensor imaging tractography atlas for virtual in vivo dissections. Cortex 44(8):1105-1132. https://doi.org/10.1016/j.cortex.2008.05.004

Conrad CD, Stumpf WE (1975) Direct visual input to the limbic system: crossed retinal projections to the nucleus anterodorsalis thalami in the tree shrew. Exp Brain Res. https://doi. org/10.1007/bf00235456

Ding SL (2013) Comparative anatomy of the prosubiculum, subiculum, presubiculum, postsubiculum, and parasubiculum in human, monkey, and rodent. J Comp Neurol 521(18):4145-4162. https:// doi.org/10.1002/cne. 23416

Ding S-L, Morecraft RJ, Van Hoesen GW (2003) Topography, cytoarchitecture, and cellular phenotypes of cortical areas that form the cingulo-parahippocampal isthmus and adjoining retrocalcarine areas in the monkey. J Comp Neurol 456(2):184-201. https://doi. org/10.1002/cne.10516

Ding S-LL, Royall JJ, Sunkin SM, Ng L, Facer BA, Lesnar P, Guillozet-Bongaarts A, McMurray B, Szafer A, Dolbeare TA, Stevens A, Tirrell L, Benner T, Caldejon S, Dalley RA, Dee N, Lau C, Nyhus J, Reding M, Riley ZL, Sandman D, Shen E, van der Kouwe A, Varjabedian A, Write M, Zollei L, Dang C, Knowles JA, Koch C, Phillips JW, Sestan N, Wohnoutka P, Zielke HR, Hohmann JG, Jones AR, Bernard A, Hawrylycz MJ, Hof PR, Fischl B, Lein ES (2016) Comprehensive cellular-resolution atlas of the adult human brain. J Comp Neurol 524(16):3127-3481. https ://doi.org/10.1002/cne.24080

Elshout JA, van den Berg AV, Haak KV (2018) Human V2A: A map of the peripheral visual hemifield with functional connections to scene-selective cortex. J Vis 18(9):22. https://doi. org/10.1167/18.9.22

Falchier A, Schroeder CE, Hackett TA, Lakatos P, Nascimento-Silva S, Ulbert I, Karmos G, Smiley JF (2010) Projection from visual areas V2 and prostriata to caudal auditory cortex in the monkey. Cerebral Cortex 20(7):1529-1538. https://doi.org/10.1093/cerco r/bhp213 
Fischl B, Sereno MI, Dale AM (1999) Cortical surface-based analysis: II: inflation, flattening, and a surface-based coordinate system. Neuroimage 9(2):195-207

Florence SL, Casagrande VA (1987) Organization of individual afferent axons in layer IV of striate cortex in a primate. J Neurosci 7(12):3850-3868

Glasser MF, Van Essen DC (2011) Mapping human cortical areas in vivo based on myelin content as revealed by T1- and T2-weighted MRI. J Neurosci 31(32):11597-11616. https://doi. org/10.1523/JNEUROSCI.2180-11.2011

Glasser MF, Coalson TS, Robinson EC, Hacker CD, Harwell J, Yacoub E, Ugurbil K, Andersson J, Beckmann CF, Jenkinson M, Smith SM, Essen DC (2016) A multi-modal parcellation of human cerebral cortex. Nature 536(7615):171-178. https://doi.org/10.1038/ nature 18933

Glasser MF, Sotiropoulos SN, Wilson JA, Coalson TS, Fischl B, Andersson JL, Xu J, Jbabdi S, Webster M, Polimeni JR, Van Essen DC, Jenkinson M, Consortium WU-MH (2013) The minimal preprocessing pipelines for the Human Connectome Project. Neuroimage 80:105-124. https://doi.org/10.1016/j.neuro image.2013.04.127

Goga C, Ture U (2015) The anatomy of Meyer's loop revisited: changing the anatomical paradigm of the temporal loop based on evidence from fiber microdissection. J Neurosurg 122(6):1253-1262. https://doi.org/10.3171/2014.12.JNS14281

Greco V, Frijia F, Mikellidou K, Montanaro D, Farini A, D’Uva M, Poggi P, Pucci M, Sordini A, Morrone MC, Burr DC (2016) A low-cost and versatile system for projecting wide-field visual stimuli within fMRI scanners. Behav Res Methods 48:614-620. https://doi.org/10.3758/s13428-015-0605-0

Gunaydin M, Ozsahin F (2018) Transient visual loss: transient lesion in the splenium of the corpus callosum. Turk J Emerg Med 18(3):128-130. https://doi.org/10.1016/j.tjem.2017.12.004

Hagiwara A, Hori M, Kamagata K, Warntjes M, Matsuyoshi D, Nakazawa M, Ueda R, Andica C, Koshino S, Maekawa T, Irie R, Takamura T, Kumamaru KK, Abe O, Aoki S (2018) Myelin measurement: comparison between simultaneous tissue relaxometry, magnetization transfer saturation index, and $\mathrm{T} 1 \mathrm{w} / \mathrm{T} 2 \mathrm{w}$ ratio methods. Sci Rep 8(1):10554. https://doi.org/10.1038/ s41598-018-28852-6

Mai JK, Assheuer J, Paxinos G (2004) Atlas of the human brain, 4th edn. Elsevier Academic Press, Boston

Kalyvas A, Koutsarnakis C, Komaitis S, Karavasilis E, Christidi F, Skandalakis GP, Liouta E, Papakonstantinou O, Kelekis N, Duffau H, Stranjalis G (2019) Mapping the human middle longitudinal fasciculus through a focused anatomo-imaging study: shifting the paradigm of its segmentation and connectivity pattern. Brain Struct Funct. https://doi.org/10.1007/s00429-019-01987-6

Kobayashi Y, Amaral DG (2003) Macaque monkey retrosplenial cortex: II. Cortical afferents. J Comp Neurol 466:48-79. https://doi. org/10.1002/cne.10883

Leong JK, Pestilli F, Wu CC, Samanez-Larkin GR, Knutson B (2016) White-matter tract connecting anterior insula to nucleus accumbens correlates with reduced preference for positively skewed gambles. Neuron 89(1):63-69. https://doi.org/10.1016/j.neuro n.2015.12.015

Liu T, Pestilli F, Carrasco M (2005) Transient attention enhances perceptual performance and FMRI response in human visual cortex. Neuron 45(3):469-477. https://doi.org/10.1016/j.neuro n.2004.12.039

Lu W, Chen S, Chen X, Hu J, Xuan A, Ding SL (2019) Localization of area prostriata and its connections with primary visual cortex in rodent. J Comp Neurol. https://doi.org/10.1002/cne.24760

Maier-Hein KH, Neher PF, Houde JC, Cote MA, Garyfallidis E, Zhong J, Chamberland M, Yeh FC, Lin YC, Ji Q, Reddick WE, Glass JO, Chen DQ, Feng Y, Gao C, Wu Y, Ma J, Renjie H, Li Q, Westin
CF, Deslauriers-Gauthier S, Gonzalez JOO, Paquette M, St-Jean S, Girard G, Rheault F, Sidhu J, Tax CMW, Guo F, Mesri HY, David S, Froeling M, Heemskerk AM, Leemans A, Bore A, Pinsard B, Bedetti C, Desrosiers M, Brambati S, Doyon J, Sarica A, Vasta R, Cerasa A, Quattrone A, Yeatman J, Khan AR, Hodges W, Alexander S, Romascano D, Barakovic M, Auria A, Esteban O, Lemkaddem A, Thiran JP, Cetingul HE, Odry BL, Mailhe B, Nadar MS, Pizzagalli F, Prasad G, Villalon-Reina JE, Galvis J, Thompson PM, Requejo FS, Laguna PL, Lacerda LM, Barrett R, Dell'Acqua F, Catani M, Petit L, Caruyer E, Daducci A, Dyrby TB, Holland-Letz T, Hilgetag CC, Stieltjes B, Descoteaux M (2017) The challenge of mapping the human connectome based on diffusion tractography. Nat Commun 8(1):1349. https://doi. org/10.1038/s41467-017-01285-x

Martino J, da Silva-Freitas R, Caballero H, Marco de Lucas E, Garcia-Porrero JA, Vazquez-Barquero A (2013) Fiber dissection and diffusion tensor imaging tractography study of the temporoparietal fiber intersection area. Neurosurgery 72 (1 Suppl Operative):87-97; (discussion 97-88). https://doi.org/10.1227/ NEU.0b013e318274294b

Mikellidou K, Arrighi R, Aghakhanyan G, Tinelli F, Frijia F, Crespi S, Masi DF, Montanaro D, Morrone MC (2017a) Plasticity of the human visual brain after early cortical lesion. Neuropsychologia. https://doi.org/10.1016/j.neuropsychologia.2017.10.033

Mikellidou K, Kurzawski JW, Frijia F, Montanaro D, Greco V, Morrone MC, Burr DC (2017b) Area prostriata in the human brain. Curr Biol 27(19):3056-3060. https://doi.org/10.1016/j. cub.2017.08.065

Morecraft RJ, Rockland KS, Van Hoesen GW (2000) Localization of area prostriata and its projection to the cingulate motor cortex in the rhesus monkey. Cereb Cortex 10(2):192-203. https://doi. org/10.1093/cercor/10.2.192

Onufrowicz W (1887) Das balkenlose Mikrocephalengehirn Hofmann. Archiv für Psychiatrie und Nervenkrankheiten 18(2):305-328. https://doi.org/10.1007/bf02054370

Palmer SM, Rosa MGP (2006) A distinct anatomical network of cortical areas for analysis of motion in far peripheral vision. Eur J Neurosci 24(8):2389-2405. https://doi.org/10.111 $1 /$ j.1460-9568.2006.05113.x

Parraga RG, Ribas GC, Welling LC, Alves RV, de Oliveira E (2012) Microsurgical anatomy of the optic radiation and related fibers in 3-dimensional images. Neurosurgery 71 (1 Suppl Operative):160-171 (discussion 171-162). https://doi.org/10.1227/ NEU.0b013e3182556fde

Pestilli F (2015) Test-retest measurements and digital validation for in vivo neuroscience. Sci Data 2:140057. https://doi.org/10.1038/ sdata. 2014.57

Pestilli F (2018) Human white matter and knowledge representation. PLoS Biol 16(4):e2005758. https://doi.org/10.1371/journ al.pbio. 2005758

Pestilli F, Yeatman JD, Rokem A, Kay KN, Wandell BA (2014) Evaluation and statistical inference for human connectomes. Nat Methods 11(10):1058-1063. https://doi.org/10.1038/nmeth.3098

Pierpaoli C, Jezzard P, Basser PJ, Barnett A, Di Chiro G (1996) Diffusion tensor MR imaging of the human brain. Radiology 201(3):637-648. https://doi.org/10.1148/radiology.201.3.8939209

Pierpaoli C, Barnett A, Pajevic S, Chen R, Penix LR, Virta A, Basser $P$ (2001) Water diffusion changes in Wallerian degeneration and their dependence on white matter architecture. Neuroimage 13(6 Pt 1):1174-1185. https://doi.org/10.1006/nimg.2001.0765

Puzniak RJ, Ahmadi K, Kaufmann J, Gouws A, Morland AB, Pestilli F, Hoffmann MB (2019) Quantifying nerve decussation abnormalities in the optic chiasm. Neuroimage Clin 24:102055. https://doi. org/10.1016/j.nicl.2019.102055

Robinson EC, Jbabdi S, Glasser MF, Andersson J, Burgess GC, Harms MP, Smith SM, Van Essen DC, Jenkinson M (2014) MSM: a new 
flexible framework for multimodal surface matching. Neuroimage 100:414-426. https://doi.org/10.1016/j.neuroimage.2014.05.069

Robinson EC, Garcia K, Glasser MF, Chen Z, Coalson TS, Makropoulos A, Bozek J, Wright R, Schuh A, Webster M, Hutter J, Price A, Cordero Grande L, Hughes E, Tusor N, Bayly PV, Van Essen DC, Smith SM, Edwards AD, Hajnal J, Jenkinson M, Glocker B, Rueckert D (2018) Multimodal surface matching with higherorder smoothness constraints. Neuroimage 167:453-465. https:// doi.org/10.1016/j.neuroimage.2017.10.037

Rockland KS (2012) Visual system: prostriata-a visual area off the beaten path. Curr Biol 22(14):571-573. https://doi.org/10.1016/j. cub.2012.05.030

Rokem A, Takemura H, Bock AS, Scherf KS, Behrmann M, Wandell BA, Fine I, Bridge H, Pestilli F (2017) The visual white matter: the application of diffusion MRI and fiber tractography to vision science. J Vis 17(2):4. https://doi.org/10.1167/17.2.4

Rosa MG, Casagrande VA, Preuss T, Kaas JH (1997) Visual field representation in striate and prestriate cortices of a prosimian primate (Galago garnetti). J Neurophysiol 77(6):3193-3217. https://doi. org/10.1152/jn.1997.77.6.3193

Rossini PM, Di Iorio R, Bentivoglio M, Bertini G, Ferreri F, Gerloff C, Ilmoniemi RJ, Miraglia F, Nitsche MA, Pestilli F, Rosanova M, Shirota Y, Tesoriero C, Ugawa Y, Vecchio F, Ziemann U, Hallett M (2019) Methods for analysis of brain connectivity: an IFCNsponsored review. Clin Neurophysiol 130(10):1833-1858. https ://doi.org/10.1016/j.clinph.2019.06.006

Rykhlevskaia E, Uddin LQ, Kondos L, Menon V (2009) Neuroanatomical correlates of developmental dyscalculia: combined evidence from morphometry and tractography. Front Hum Neurosci 3:51. https://doi.org/10.3389/neuro.09.051.2009

Sani I, McPherson BC, Stemmann H, Pestilli F, Freiwald WA (2019) Functionally defined white matter of the macaque monkey brain reveals a dorso-ventral attention network. Elife. https://doi. org/10.7554/eLife.40520

Sanides F (1969) Comparative architectonics of the neocortex of mammals and their evolutionary interpretation. Ann N Y Acad Sci 167(1):404-423. https://doi.org/10.1111/j.1749-6632.1969.tb204 59. $\mathrm{x}$

Sanides F, Vitzthum HG (1965) Zur Architektonik der menschlichen Sehrinde und den Prinzipien ihrer Entwicklung. Deutsche Zeitschrift f Nervenheilkunde 187(7):680-707. https://doi. org/10.1007/BF00243937

Schmahmann JD, Pandya DN (2007) The complex history of the fronto-occipital fasciculus. J Hist Neurosci 16(4):362-377. https ://doi.org/10.1080/09647040600620468

Shams Z, Norris DG, Marques JP (2019) A comparison of in vivo MRI based cortical myelin mapping using T1w/T2w and R1 mapping at 3T. PLoS ONE 14(7):e0218089. https://doi.org/10.1371/journ al.pone.0218089

Sherman SM, Guillery RW (2002) The role of the thalamus in the flow of information to the cortex. Philos Trans R Soc Lond B Biol Sci 357(1428):1695-1708. https://doi.org/10.1098/rstb.2002.1161

Smith RE, Tournier JD, Calamante F, Connelly A (2012) Anatomically-constrained tractography: improved diffusion MRI streamlines tractography through effective use of anatomical information. Neuroimage 62(3):1924-1938. https://doi.org/10.1016/j. neuroimage.2012.06.005

Takemura H, Caiafa CF, Wandell BA, Pestilli F (2016a) Ensemble tractography. PLoS Comput Biol 12(2):e1004692. https://doi. org/10.1371/journal.pcbi.1004692

Takemura H, Rokem A, Winawer J, Yeatman JD, Wandell BA, Pestilli F (2016b) A major human white matter pathway between dorsal and ventral visual cortex. Cereb Cortex 26(5):2205-2214. https ://doi.org/10.1093/cercor/bhv064

Takemura H, Pestilli F, Weiner KS, Keliris GA, Landi SM, Sliwa J, Ye FQ, Barnett MA, Leopold DA, Freiwald WA, Logothetis NK,
Wandell BA (2017) Occipital white matter tracts in human and macaque. Cereb Cortex 27(6):3346-3359. https://doi.org/10.1093/ cercor/bhx070

Takemura H, Pestilli F, Weiner KS (2019) Comparative neuroanatomy: Integrating classic and modern methods to understand association fibers connecting dorsal and ventral visual cortex. Neurosci Res 146:1-12. https://doi.org/10.1016/j.neures.2018.10.011

Tax CM, Duits R, Vilanova A, ter Haar Romeny BM, Hofman P, Wagner L, Leemans A, Ossenblok P (2014) Evaluating contextual processing in diffusion MRI: application to optic radiation reconstruction for epilepsy surgery. PLoS ONE 9(7):e101524. https:// doi.org/10.1371/journal.pone.0101524

Thomas C, Ye FQ, Irfanoglu MO, Modi P, Saleem KS, Leopold DA, Pierpaoli C (2014) Anatomical accuracy of brain connections derived from diffusion MRI tractography is inherently limited. Proc Natl Acad Sci U S A 111(46):16574-16579. https://doi.org/10.1073/ pnas. 1405672111

Tournier JD, Calamante F, Gadian DG, Connelly A (2004) Direct estimation of the fiber orientation density function from diffusion-weighted MRI data using spherical deconvolution. Neuroimage 23(3):1176-1185. https://doi.org/10.1016/j.neuroimage .2004.07.037

Tournier JD, Smith R, Raffelt D, Tabbara R, Dhollander T, Pietsch M, Christiaens D, Jeurissen B, Yeh CH, Connelly A (2019) MRtrix3: A fast, flexible and open software framework for medical image processing and visualisation. Neuroimage. https://doi. org/10.1016/j.neuroimage.2019.116137

Van Essen DC, Smith SM, Barch DM, Behrens TE, Yacoub E, Ugurbil K, Consortium WU-MH (2013) The WU-Minn Human Connectome Project: an overview. Neuroimage 80:62-79. https://doi. org/10.1016/j.neuroimage.2013.05.041

Veltin A (2003) The "Rhapsodies on the use of the psychological treatment method for mental disorders" by Johann Christian Reil, Halle, 1803. Psychiatr Prax 30(5):288-289

Wandell BA (2016) Clarifying human white matter. Annu Rev Neurosci 39:103-128. https://doi.org/10.1146/annurev-neuro-07081 5-013815

Wandell BA, Winawer J (2015) Computational neuroimaging and population receptive fields. Trends Cogn Sci 19(6):349-357. https:// doi.org/10.1016/j.tics.2015.03.009

Wandell BA, Brewer AA, Dougherty RF (2005) Visual field map clusters in human cortex. Philos Trans R Soc Lond B Biol Sci 360(1456):693-707. https://doi.org/10.1098/rstb.2005.1628

Yakar F, Eroglu U, Peker E, Armagan E, Comert A, Ugur HC (2018) Structure of corona radiata and tapetum fibers in ventricular surgery. J Clin Neurosci. https://doi.org/10.1016/j.jocn.2018.08.041

Yeatman JD, Dougherty RF, Myall NJ, Wandell BA, Feldman HM (2012) Tract profiles of white matter properties: automating fiber-tract quantification. PLoS ONE 7(11):e49790. https://doi. org/10.1371/journal.pone.0049790

Yeatman JD, Weiner KS, Pestilli F, Rokem A, Mezer A, Wandell BA (2014) The vertical occipital fasciculus: a century of controversy resolved by in vivo measurements. Proc Natl Acad Sci U S A 111(48):E5214-5223. https://doi.org/10.1073/pnas.1418503111

Yoshimine S, Ogawa S, Horiguchi H, Terao M, Miyazaki A, Matsumoto K, Tsuneoka H, Nakano T, Masuda Y, Pestilli F (2018) Age-related macular degeneration affects the optic radiation white matter projecting to locations of retinal damage. Brain Struct Funct. https:// doi.org/10.1007/s00429-018-1702-5

Yu HH, Chaplin TA, Davies AJ, Verma R, Rosa MGP (2012) A specialized area in limbic cortex for fast analysis of peripheral vision. Curr Biol 22(14):1351-1357. https://doi.org/10.1016/j. cub.2012.05.029

Zhang Y, Zhang J, Oishi K, Faria AV, Jiang H, Li X, Akhter K, Rosa-Neto P, Pike GB, Evans A, Toga AW, Woods R, Mazziotta JC, Miller MI, van Zijl PC, Mori S (2010) Atlas-guided tract 
reconstruction for automated and comprehensive examination of the white matter anatomy. Neuroimage 52(4):1289-1301. https:// doi.org/10.1016/j.neuroimage.2010.05.049
Publisher's Note Springer Nature remains neutral with regard to jurisdictional claims in published maps and institutional affiliations. 